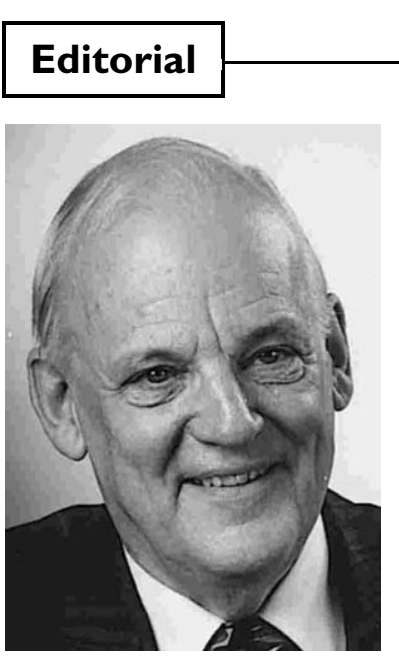

Gösta Samuelson

Editor-in-chief gosta.samuelson@htu.se

\title{
What to eat and not to eat: still a question of debate
}

Obesity and overweight are a huge concern in the whole world today. In April 2003 the Food and Agricultural Organization (FAO) and the World Health Organization (WHO) presented the report "Diet, nutrition and the prevention of chronic diseases" (WHO Technical Report Series No. 916). Dietary factors such as foods rich in fat and/or sugar, sugar-sweetened soft drinks and a sedentary lifestyle are focused on in relation to weight gain and obesity. Regular physical activity and a high intake of dietary fibre, and possibly also foods with a low glycaemic index, may decrease the risk. The report is a valuable compilation of global consumption patterns and trends as a basis for nutrient intake goals to prevent diet-related chronic diseases. However, a discussion among scientists has emerged about the role of fat in overweight and obesity. In this issue of Scandinavian Journal of Nutrition (SJN), Walter Willett and Arne Astrup present their views - in part different, in part concordant - of the scientific evidence behind the association between dietary fat and obesity.

\section{Dietary fat and obesity: current opinion}

Many cross-sectional studies show a correlation between dietary fat and body fatness, whereas others do not. It is important in this context to remember the limitations of epidemiological studies in proving cause-effect relationship and the potential of confounders. Randomized short-term studies of obesity treatment have shown a weight loss of about $1-4 \mathrm{~kg}$ when $10-15 E \%$ from fat is replaced by carbohydrates. However, Willett's opinion is that fat consumption in the range of $18-40 \%$ of energy has little effect on body fat. From a review of current scientific publications, he concludes that long-term randomized studies are few and the results are disappointing. Dietary protein content and the glycaemic index of carbohydrates may be more important than fat intake.

In contrast, Astrup argues in favour of the importance of decreasing the amount of fat intake and the importance of the higher energy density of fatty foods. He also underlines the role of sugar-rich beverages as an energy source, the consumption of which has increased tremendously during the past few decades. He concludes that the consumption of diets with reduced fat content, and high in protein and dietary fibre, contributes significantly to the prevention of weight gain in normal-weight subjects and causes a weight loss of about 3-4 kg in overweight subjects in a relatively short period. Both authors agree that abundant evidence supports physical activity as being at least as important as nutritional factors, and agree on the need for prospective, longterm, well-designed, randomized studies on the relation between dietary components and obesity before any definite conclusions can be drawn.

\section{Rebuilding the food pyramid}

In the USA, Willett and Stampfer have proposed that the well-known food pyramid should be reconstructed (Scientific American 2003; 203: 6471). In brief, the new pyramid recommends a base of physical activity, healthy fats, nuts and wholegrain foods, vegetables and fruits, and at the top, less intake of refined carbohydrates, butter, potatoes, red meat and pasta. The proposal is based on epidemiological studies, and needs confirmation by intervention studies. Comments on the new pyramid are welcomed for forthcoming issues of SJN.

\section{Nutrition and the brain}

In the present and the next issue of $\mathrm{SJN}$, articles are presented about the effects of nutrients on brain development and behaviour. Alexandra Richardson reviews unsaturated fatty acids and behavioural functions. She claims that the ratio of $n-6$ to $n-3$ fatty acids in the Western diet has increased dramatically. In countries like Sweden, however, where people consume plenty of spreads based on rapeseed oil and also fish, the ratio is about 5 . 